\title{
Reduce Correlated Noise in EELS Spectrum with High Quality Dark Reference
}

\author{
Vincent D.-H. Hou \\ TEM Lab, Micron Technology, Inc., 8000 S. Federal Way, P.O. Box 6, Boise, ID 83707, USA
}

Recently, a binned gain averaging (BGA) technique has been developed to suppress correlated or systematic noise in EELS spectra acquired by an array detector ${ }^{[1]}$. The authors have shown that the origin of such noise is due to incomplete correction of detector gains. In addition, the effect of dark count subtraction is addressed briefly, but not explicitly. It will be shown here that the quality of the dark reference plays is equally as important as gain correction. Such correlated noise can be suppressed with a high quality dark reference without the need of gain-averaging.

EELS spectra from a $200 \mathrm{kV}$ primary electron beam were acquired with a GIF Tridiem on an FE $\mathrm{S} / \mathrm{TEM}$ operated in STEM mode. Data was acquired with the Gatan's Spectrum Imaging package. Spectra were read out in the frame transfer mode with $2 \mathrm{~ms}$ exposure time and 128x on-chip binning in a nondispersion direction. The first spectrum image (SI) data set (Fig. 1a) was acquired with the default gain-normalization (DF) routine. Roughly halfway through the acquisition, a drift tube scan ${ }^{[2]}$ was activated so this part of the data could be processed later for gain-averaging. It is noted that each row of this SI represented a single spectrum. A portion of the data set corresponding to background noise is shown in Fig.1b. The correlated noise can easily be visualized as vertical streaks. Moreover, comparing the spectrum noise of a 2-row average to that of a 128-row average (Fib. 1c) shows background noises are indeed strongly correlated. Results of gain-averaging are shown in Fig. 2, where the zero-loss peak (ZLP) is used as an alignment reference. Streaks shown in Fig. 1b now become slanted (Fig. 2b). Consequently, the correlated noise is suppressed when multiple spectra are averaged (Fig. 2c). This result validates the effectiveness of the BGA technique.

The same experiment was repeated, but spectra were acquired in an unprocessed mode. They were processed later with manual dark and gain references. The most critical aspect of the manual dark reference is that it is an average of multiple readouts, as opposed to a single readout used by a DF routine. The contrast is clearly shown in Fig. 3a, and it can be envisaged that the correlated noise may arise from a single and noisy dark reference. The manual gain reference is also an average of multiple readouts (Fig. 3b). The manually processed results are shown in Figs. 4 and 5, which are in the same format as Figs. 1 and 2, respectively. It is clearly seen that the background noises are no longer correlated when a high quality dark reference is used. The same data processed with a manual dark reference, but a standard gain reference, shows comparable results.

Several selected signal-to-noise (SNR) ratios from this work are summarized in Tab. 1. They are calculated using the method in [1]. It is apparent that SNR improvement by use of a high quality dark reference is comparable (or better) to that of the BGA technique. An even better SNR can be achieved by employing both a high quality dark reference and the gain-averaging technique. The method of using a high quality dark reference with a standard gain reference shown in this work has an advantage over the BGA method because it eliminates the need of in situ drift-tube scanning and ex situ spectrum alignments.

The author would like to thank Dr. Boseman for providing additional details on the BGA technique.

\section{Reference}

[1] M. Boseman and V. J. Keast, Ultramicroscopy, 108 (2008) 837.

[2] V. D.-H. Hou, "Drift Tube Scan.s”, DigitalMicrograph script. 

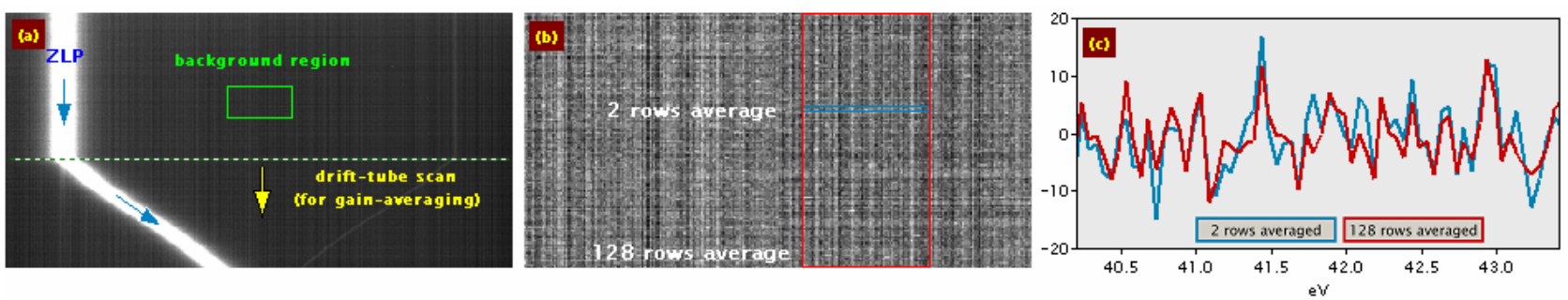

Fig. 1. PEELS spectra of zero loss peak. Dark subtraction and gain correction are handled automatically by default routine in DigitalMicrograph (a) Result shown as Spectrum Image, where each row represents a single spectrum. (b) Portion of SI denoted as background region in (a). (c) 2 and 128 rows averaged spectrum in regions marked in (b).

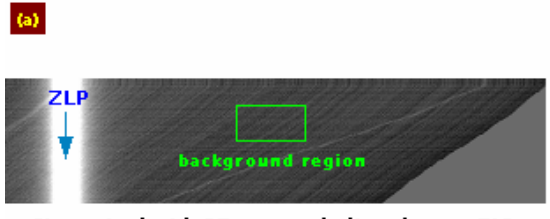

SI acquired with DT scan and aligned w.r.t. ZLP
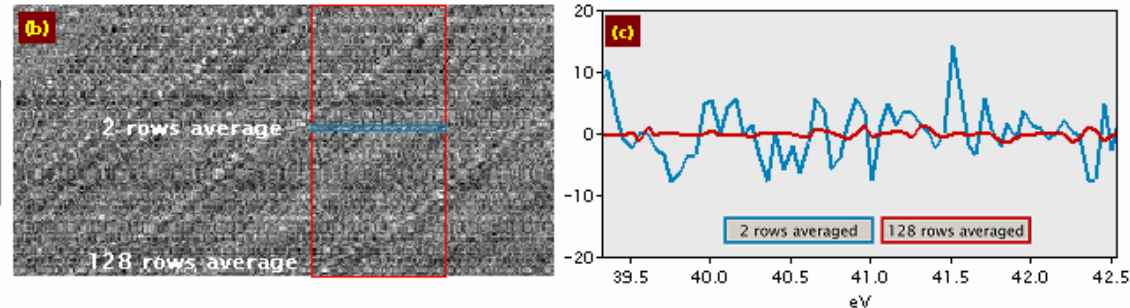

Fig. 2. Portion of PEELS spectra in Fig.1a where drift tube scan was applied during acquisition. Gain-averaging is achieved bv summing multiple rows of spectra. which were aligned first with each other with ZLP as reference.

\begin{tabular}{|c|c|c|}
\hline $\begin{array}{c}\text { SNR = ZLP.P peak intensity / std. dev. of } \\
\text { selected background region }\end{array}$ & $\begin{array}{c}\text { SNR } \\
\text { (128 spectra sum) }\end{array}$ \\
\hline \hline $\begin{array}{c}\text { Default } \\
\text { dark \& gain }\end{array}$ & direct & 5,800 \\
\hline $\begin{array}{c}\text { Manual } \\
\text { dark \& gain }\end{array}$ & gain-averaging & 39,300 \\
\hline
\end{tabular}

Table 1 SNR of EELS spectra acquired and processed by various methods.
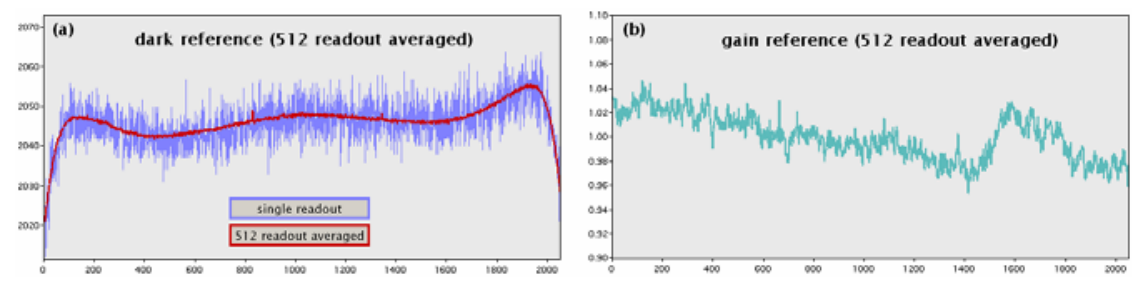

Fig. 3. Dark and gain reference used for manual spectrum processing. Both references are acquired manually and are averages of 512 references.
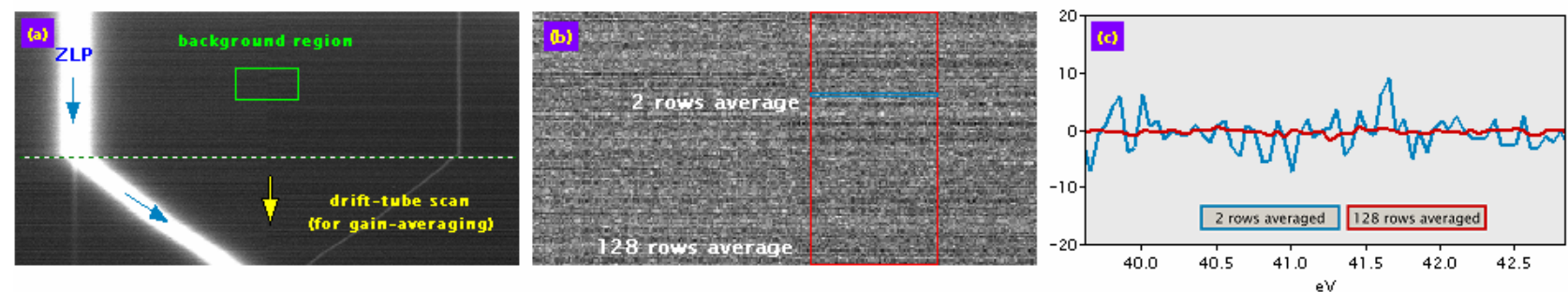

Fig. 4. PEELS spectrum image and spectra similar to those shown in Fig. 1, but data is acquired in "unprocessed" mode. Dark subtraction and gain correction are processed manually using references shown in Fig. 3.
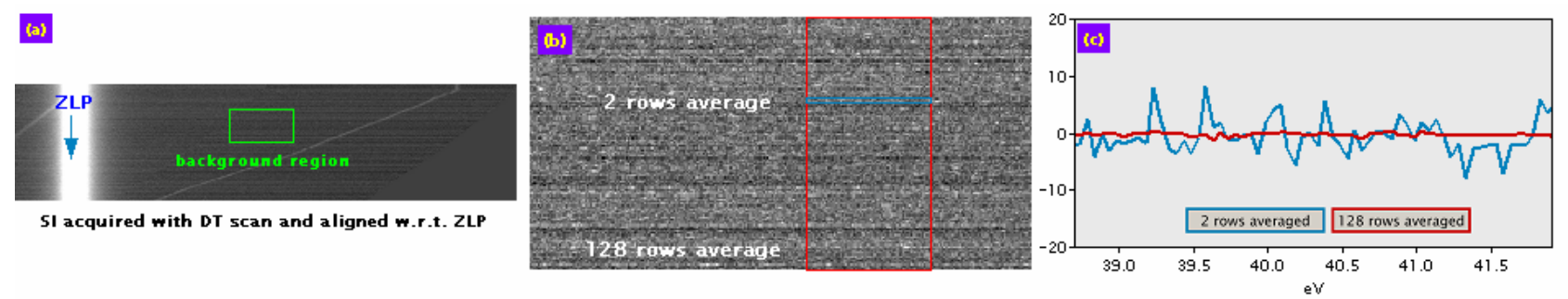

Fig. 5. Portion of PEELS spectra in Fig.4a where drift tube scan was applied during acquisition. Gain-averaging is achieved by summing multiple rows of spectra, which were aligned first with each other with ZLP as reference. 